\title{
Spin/Orbital Structure of the Nucleon from Wigner Distributions
}

\author{
B. Pasquini \\ Dipartimento di Fisica, Università degli Studi di Pavia, Pavia, Italy \\ Istituto Nazionale di Fisica Nucleare, Sezione di Pavia, Pavia, Italy \\ pasquini@pv.infn.it \\ C. Lorcé \\ SLAC National Accelerator Laboratory, Stanford University, Menlo Park, \\ California 94025 USA, IFPA, AGO Department, Université de Liège, \\ Sart-Tilman, 4000 Liège, Belgium \\ c.lorce@ulg.ac.be \\ Published 29 February 2016
}

\begin{abstract}
We present results for the Wigner distributions of the nucleon, which provide multidimensional images of the quark distributions in the phase space and can be seen as the mother distributions of the standard generalized and transverse-momentum dependent parton distributions. We discuss the general features of these distributions within a lightfront constituent quark model, emphasizing the new information that can be deduced about the spin-orbit correlations of the quarks in the nucleon.
\end{abstract}

Keywords: Wigner distributions; light-front quark models.

PACS numbers:12.38. - t, 12.39.-x,14.20.Dh

\section{Introduction}

A new way to explore the parton distributions in the nucleon has recently been introduced through the Wigner-type quark and gluon distributions. ${ }^{1,2}$ These functions have a direct connection with the generalized parton correlation functions (GPCFs) which were recently introduced in Refs. 3, 4 and further explored in Refs. 5, 6. The GPCFs are the distributions that parametrize the fully unintegrated, off-diagonal quark-quark correlator for a hadron. In the case of the nucleon and after integration over the light-cone energy of the quark, one finds the so-called generalized transverse-momentum dependent parton distributions (GTMDs). At leading-twist there are 16 GTMDs which depend on the light-cone three-momentum

This is an Open Access article published by World Scientific Publishing Company. It is distributed under the terms of the Creative Commons Attribution 3.0 (CC-BY) License. Further distribution of this work is permitted, provided the original work is properly cited. 


\section{B. Pasquini \& C. Lorcé}

of the quark and, in addition, on the momentum transfer to the nucleon $\Delta^{\mu}$. After two-dimensional Fourier transform from $\vec{\Delta}_{\perp}$ to the impact-parameter space coordinates $\vec{b}_{\perp}$, in a frame without momentum transfer along the light-cone direction, one obtains the Wigner distributions which are completely consistent with special relativity. Although we did not yet identify a proper high-energy process to access these functions, it is important to note that, in general, there exists no argument according to which, as a matter of principle, GTMDs cannot be measured. In this context, model studies assume a crucial role to disclose which kind of information are encoded in these functions. First model studies have been performed within a relativistic light-front constituent quark model (LFCQM), ${ }^{7}$ and afterwards have been also presented in a scalar diquark model of the nucleon, ${ }^{8}$ in the quarktarget model, ${ }^{8-10}$ in perturbative QCD for large transverse parton momenta ${ }^{8}$ and in a light-front spectator model. ${ }^{11}$ Here we review a few results obtained within a LFCQM.

\section{Wigner Distributions}

Following Refs. 1, 2, we define the Hermitian Wigner operators for quarks at a fixed light-cone time $z^{+}=0$ as follows ${ }^{\mathrm{a}}$

$$
\left.\widehat{W}^{[\Gamma]}\left(\vec{b}_{\perp}, \vec{k}_{\perp}, x\right) \equiv \frac{1}{2} \int \frac{\mathrm{d} z^{-} \mathrm{d}^{2} \vec{z}_{\perp}}{(2 \pi)^{3}} e^{i\left(x p^{+} z^{-}-\vec{k}_{\perp} \cdot \vec{z}_{\perp}\right)} \bar{\psi}\left(y-\frac{z}{2}\right) \Gamma \mathcal{W} \psi\left(y+\frac{z}{2}\right)\right|_{z^{+}=0}
$$

with $y^{\mu}=\left[0,0, \vec{b}_{\perp}\right], p^{+}$the average nucleon longitudinal momentum and $x=k^{+} / p^{+}$ the average fraction of nucleon longitudinal momentum carried by the active quark. The superscript $\Gamma$ stands for any twist-two Dirac operator $\Gamma=\gamma^{+}, \gamma^{+} \gamma_{5}, i \sigma^{j+} \gamma_{5}$ with $j=1,2$. A Wilson line $\mathcal{W} \equiv \mathcal{W}\left(y-\frac{z}{2}, y+\frac{z}{2} \mid n\right)$ ensures the color-gauge invariance of the Wigner operator, connecting the points $\left(y-\frac{z}{2}\right)$ and $\left(y+\frac{z}{2}\right)$. Note that $\vec{b}_{\perp}$ and $\vec{k}_{\perp}$ are not Fourier conjugate variables, like in the usual quantum-mechanical Wigner distributions. Rather, if $\vec{r}_{i \perp}\left(\vec{r}_{f}\right)$ and $\vec{k}_{i \perp}\left(\vec{k}_{f \perp}\right)$ are the transverse position and momentum coordinates of the initial (final) quark operator $\psi(\bar{\psi})$, one sees that the average quark momentum $\vec{k}_{\perp}=\frac{\vec{k}_{f \perp}+\vec{k}_{i \perp}}{2}$ is the Fourier conjugate variable of the quark displacement $\vec{z}_{\perp}=\vec{r}_{i \perp}-\vec{r}_{f \perp}$ and the momentum transfer to the quark $\vec{\Delta}_{\perp}=\vec{k}_{f \perp}-\vec{k}_{i \perp}$ is the Fourier conjugate variable of the average quark position $\vec{b}_{\perp}=\frac{\vec{r}_{f \perp}+\vec{r}_{i \perp}}{2}$. This can be easily seen by expressing the argument of the Fourier exponential as $\vec{k}_{f \perp} \cdot \vec{r}_{f \perp}-\vec{k}_{i \perp} \cdot \vec{r}_{i \perp}=\vec{\Delta}_{\perp} \cdot \vec{b}_{\perp}-\vec{k}_{\perp} \cdot \vec{z}_{\perp}$. Even though $\vec{b}_{\perp}$ and $\vec{k}_{\perp}$ are not Fourier conjugate variables, they are subjected to Heisenberg's uncertainty principle because the corresponding quantum-mechanical operators do not commute $\left[\hat{\vec{b}}_{\perp}, \hat{\vec{k}}_{\perp}\right] \neq 0$.

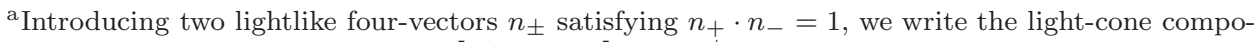
nents of a generic four-vector $a$ as $\left[a^{+}, a^{-}, \vec{a}_{\perp}\right]$ with $a^{ \pm}=a \cdot n_{\mp}$.
} 
We define the Wigner distributions in terms of the matrix elements of the Wigner operators sandwiched between nucleon states with polarization $\vec{S}$ as follows

$$
\rho^{[\Gamma]}\left(\vec{b}_{\perp}, \vec{k}_{\perp}, x, \vec{S}\right) \equiv \int \frac{\mathrm{d}^{2} \vec{\Delta}_{\perp}}{(2 \pi)^{2}}\left\langle p^{+}, \frac{\vec{\Delta}_{\perp}}{2}, \vec{S}\left|\widehat{W}^{[\Gamma]}\left(\vec{b}_{\perp}, \vec{k}_{\perp}, x\right)\right| p^{+},-\frac{\vec{\Delta}_{\perp}}{2}, \vec{S}\right\rangle .
$$

As outlined in Ref. 7, such matrix elements can be interpreted as two-dimensional Fourier transforms of GTMDs in impact-parameter space. Contrary to all the other distribution functions, the GTMDs are in general complex-valued functions. However the two-dimensional Fourier transforms of the GTMDs are always real-valued functions, in accordance with their interpretation as phase-space distributions.

\subsection{Results and discussions}

Considering all the possible polarizations of active quark and nucleon, we can have 16 configurations which can be written in terms of 16 independent combinations of the GTMDs. To keep the discussion relatively simple, we focus on cases without any transverse polarization. The Wigner distribution of quarks with longitudinal polarization $\lambda$ in a nucleon with longitudinal polarization $\Lambda$ is obtained for $\Gamma=$ $\gamma^{+} \frac{1+\lambda \gamma_{5}}{2}$ and $\vec{S}=\Lambda \vec{e}_{z}$

$$
\rho_{\Lambda \lambda}\left(\vec{b}_{\perp}, \vec{k}_{\perp}, x\right) \equiv \frac{1}{2}\left[\rho^{\left[\gamma^{+}\right]}\left(\vec{b}_{\perp}, \vec{k}_{\perp}, x, \Lambda \vec{e}_{z}\right)+\lambda \rho^{\left[\gamma^{+} \gamma_{5}\right]}\left(\vec{b}_{\perp}, \vec{k}_{\perp}, x, \Lambda \vec{e}_{z}\right)\right] .
$$

We decompose it as follows

$$
\begin{aligned}
\rho_{\Lambda \lambda}\left(\vec{b}_{\perp}, \vec{k}_{\perp}, x\right)= & \frac{1}{2}\left[\rho_{U U}\left(\vec{b}_{\perp}, \vec{k}_{\perp}, x\right)+\Lambda \rho_{L U}\left(\vec{b}_{\perp}, \vec{k}_{\perp}, x\right)+\lambda \rho_{U L}\left(\vec{b}_{\perp}, \vec{k}_{\perp}, x\right)\right. \\
& \left.+\Lambda \lambda \rho_{L L}\left(\vec{b}_{\perp}, \vec{k}_{\perp}, x\right)\right],
\end{aligned}
$$

where $\rho_{U U}$ is the Wigner distribution of unpolarized quarks in an unpolarized nucleon, $\rho_{L U}$ is the distortion due to the longitudinal polarization of the nucleon, $\rho_{U L}$ is the distortion due to the longitudinal polarization of the quarks, and $\rho_{L L}$ represents the distortion due to the correlation between quark and nucleon longitudinal polarizations.

We will discuss the results for the Wigner distributions in Eq. (4) within a LFCQM that has already been used for the description of the GPDs, ${ }^{12,13}$ the transverse-momentum dependent parton distributions (TMDs) ${ }^{14-18}$ and electroweak properties of the nucleon, ${ }^{19}$ giving a typical accuracy of about $30 \%$ in comparison with available data in the valence region. For the calculation, we use the general formalism developed in Ref. 6 for the overlap representation of the quarkquark correlator in terms of quark light-front wave-functions. Therefore, we neglect the contribution from gauge degrees of freedom, and in particular from the Wilson line in the Wigner operator (1). Beside specific features related to the quark model used for the discussion of the results, we try to sketch some general features about the behavior of the quarks in the nucleon when observed in the $\vec{b}_{\perp}$ plane at fixed $\vec{k}_{\perp}$, or in the $\vec{k}_{\perp}$ plane at fixed $\vec{b}_{\perp}$. 
We found that the Wigner distributions $\rho_{U U}$ of unpolarized quarks in an unpolarized nucleon are not axially symmetric. This deformation can be explained with naive semi-classical arguments, as a consequence of confinement which disfavors the radial motion of the quark compared to its orbital motion. Furthermore, we observed that the spread of the distributions is smaller for $u$ quarks than for $d$ quarks, especially in the $\vec{b}_{\perp}$ space, revealing that the $u$ quarks are more concentrated at the center of the proton, while the $d$-quark distribution has a tail which extends further at the periphery of the proton.

The function $\rho_{L U}$ for unpolarized quarks in a longitudinally polarized nucleon is particularly interesting because it allows us to calculate the phase-space average of the quark orbital angular momentum (OAM). ${ }^{7,20-23}$ In this way, one can relate the OAM to the GTMD $F_{1,4}$, as denoted in Ref. 3. Depending on how one chooses the path of the gauge link that makes the GTMD correlator gauge invariant, ${ }^{22,23} F_{1,4}$ provides either the (canonical) OAM of Jaffe-Manohar ${ }^{24}$ or the OAM in the definition of $\mathrm{Ji}^{25}$ (We also refer to Ref. 26 for a closely related discussion and Refs. ${ }^{27,28}$ for recent reviews on the decomposition of the nucleon spin.) The connection between OAM and $F_{1,4}$ could also make the canonical OAM accessible to Lattice QCD. ${ }^{20}$

The function $\rho_{U L}$ is related to the Fourier transform of the GTMD $G_{1,1}$. The GTMD $G_{1,1}$ can be considered as the "partner" of $F_{1,4}$ as it describes the correlation between the quark spin and the OAM in the $\hat{e}_{z}$ direction. ${ }^{7,29}$ Neither $F_{1,4}$ nor $G_{1,1}$ survive the GPD-limit nor the TMD-limit. In some sense this makes these two functions actually unique.

In the case of the distortion due to the correlation between the quark and nucleon spins, the function $\rho_{L L}$ allows us to study the distributions of the axial charge in the phase space.

Finally, taking into account all the four contributions discussed above, we can visualize the combined effects induced on the distributions by the longitudinal polarizations of the quarks and nucleon, corresponding to the Wigner distribution $\rho_{\Lambda \lambda}$. In Fig. 1, the transverse Wigner distributions of $u$ and $d$ quarks with polarization $\lambda=\uparrow, \downarrow$ in a proton with polarization $\Lambda=\uparrow$ are shown in the impact-parameter space with fixed transverse momentum $\vec{k}_{\perp}=k_{\perp} \hat{e}_{y}$ and $k_{\perp}=0.3 \mathrm{GeV}$. The deformation induced by the quark and nucleon polarizations is clearly visible in the sideway shifts of the distributions in Fig 1. In particular, when the quark and nucleon polarizations are parallel (antiparallel) the shift is in the positive (negative) $\hat{b}_{x}$ direction, see upper (lower) panels.

We learned from $\rho_{L U}$ and $\rho_{U L}$ that the $u$-quark OAM tends to be aligned with both the quark and proton polarizations. When the $u$ quark has polarization parallel to the nucleon spin, the contributions $\rho_{L U}$ and $\rho_{U L}$ interfere constructively resulting in a sideway shift in the positive $\hat{b}_{x}$ direction. When the $u$ quark has polarization antiparallel to the nucleon spin, the contributions $\rho_{L U}$ and $\rho_{U L}$ interfere destructively. Since the correlation between the OAM and the quark spin is stronger than the correlation between the OAM and the nucleon spin, it results a 

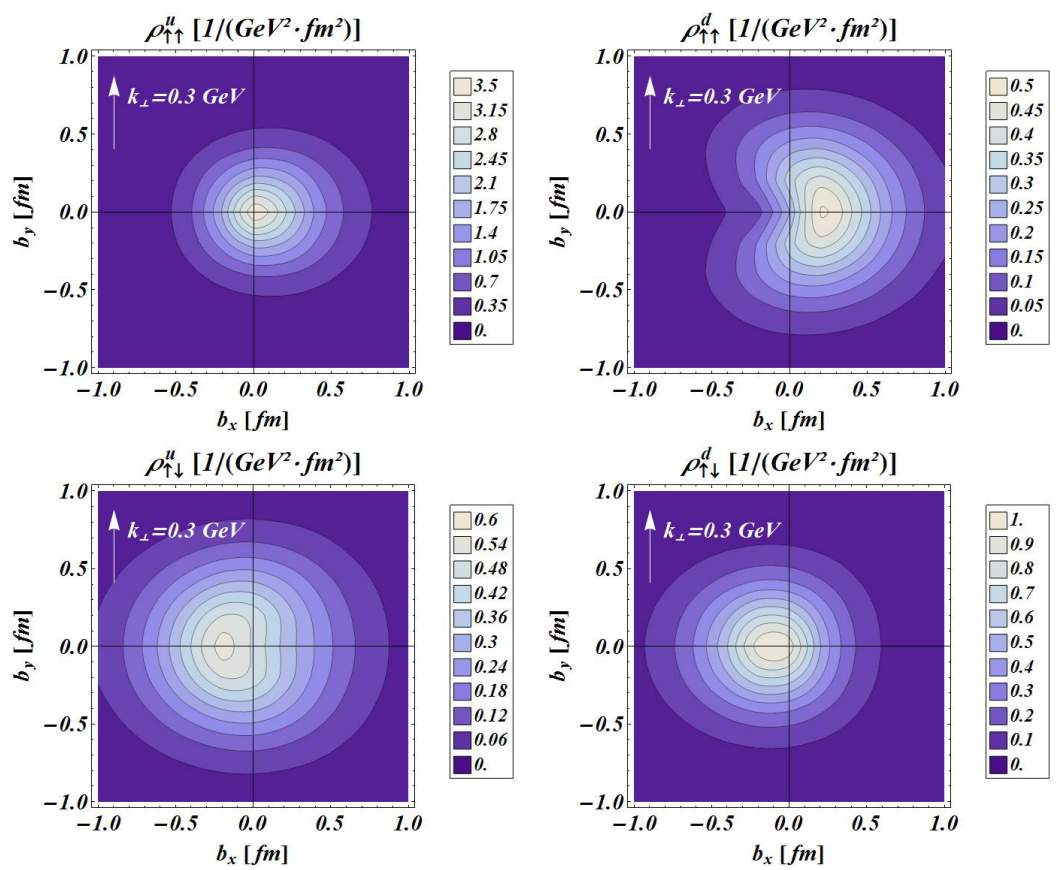

Fig. 1. The transverse Wigner distributions of longitudinally polarized quarks in a longitudinally polarized proton $(\Lambda=\uparrow$ pointing out of the plane) in impact-parameter space with fixed transverse momentum $\vec{k}_{\perp}=k_{\perp} \hat{e}_{y}$ and $k_{\perp}=0.3 \mathrm{GeV}$. Upper panels: distributions of quarks with polarization parallel to the nucleon spin $(\lambda=\uparrow)$. Lower panels: distributions of quarks with polarization antiparallel to the nucleon spin $(\lambda=\downarrow)$. The left (right) panels show the results for $u(d)$ quarks.

sideway shift in the negative $\hat{b}_{x}$ direction. For the $d$ quark, we learned from $\rho_{L U}$ and $\rho_{U L}$ that the OAM tends to be aligned with the quark polarization but antialigned with the nucleon polarization. When the $d$ quark has polarization parallel to the nucleon spin, the contributions $\rho_{L U}$ and $\rho_{U L}$ interfere destructively. Once again, the correlation between the OAM and the quark spin is stronger than the correlation between the OAM and the nucleon spin, resulting in a sideway shift in the positive $\hat{b}_{x}$ direction. When the $d$ quark has polarization antiparallel to the nucleon spin, the contributions $\rho_{L U}$ and $\rho_{U L}$ interfere constructively resulting in a sideway shift in the negative $\hat{b}_{x}$ direction.

\section{Summary}

We presented a study of the quark Wigner functions which provide the full phasespace description of the quark distributions in the nucleon. Using the light-front formalism, we derived the Wigner distributions as two-dimensional Fourier transforms of the GTMDs from the transverse-momentum transfer $\vec{\Delta}_{\perp}$ to the impact parameter $\vec{b}_{\perp}$. This derivation is not spoiled by relativistic corrections and provide us with images of the nucleon in five dimensions, namely two position and three momentum 
coordinates. We focused on the distributions of unpolarized/longitudinally polarized quarks in an unpolarized/longitudinally polarized nucleon. Furthermore, we considered only the quark contribution, neglecting all the gauge-field degrees of freedom. Beside specific features related to the quark model used for discussing the results, we tried to emphasize the physical content of the Wigner distributions. In particular, we pointed out the importance of two particular Wigner functions which neither survive the GPD-limit nor the TMD-limit and play a particular role thanks to their intimate connection to the OAM of partons. Those relations open up new opportunities to study the spin/orbital structure of the nucleon. New insights in this area could now be obtained through Lattice QCD, given the related pioneering and encouraging studies of TMDs and other parton correlation functions. ${ }^{30-32}$

\section{References}

1. X.D. Ji, Phys. Rev. Lett. 91, 062001 (2003).

2. A.V. Belitsky, X.D.. Ji and F. Yuan, Phys. Rev. D 69, 074014 (2004).

3. S. Meissner, A. Metz and M. Schlegel, JHEP 0908, 056 (2009).

4. S. Meissner, A. Metz, M. Schlegel and K. Goeke, JHEP 0808, 038 (2008).

5. C. Lorcé and B. Pasquini, JHEP 1309, 138 (2013).

6. C. Lorcé, B. Pasquini and M. Vanderhaeghen, JHEP 1105, 041 (2011).

7. C. Lorcé and B. Pasquini, Phys. Rev. D 84, 014015 (2011).

8. K. Kanazawa, C. Lorcé, A. Metz, B. Pasquini and M. Schlegel, Phys. Rev. D 90, 014028 (2014).

9. A. Mukherjee, S. Nair and V.K. Ojha, arXiv:1409.7238 [hep-ph].

10. A. Mukherjee, S. Nair and V.K. Ojha, arXiv:1501.03728 [hep-ph].

11. T. Liu and B.Q. Ma, arXiv:1501.07690 [hep-ph].

12. S. Boffi and B. Pasquini, Riv. Nuovo Cim. 30, 387 (2007).

13. B. Pasquini and S. Boffi, Phys. Lett. B 653, 23 (2007).

14. B. Pasquini, S. Cazzaniga and S. Boffi, Phys. Rev. D 78, 034025 (2008).

15. C. Lorcé and B. Pasquini, Phys. Rev. D 84, 034039 (2011).

16. S. Boffi, A.V. Efremov, B. Pasquini and P. Schweitzer, Phys. Rev. D 79, 094012 (2009).

17. B. Pasquini and F. Yuan, Phys. Rev. D 81, 114013 (2010).

18. B. Pasquini and P. Schweitzer, Phys. Rev. D 83, 114044 (2011).

19. B. Pasquini and S. Boffi, Phys. Rev. D 76, 074011 (2007).

20. Y. Hatta, Phys. Lett. B 708, 186 (2012).

21. C. Lorcé, B. Pasquini, X. Xiong and F. Yuan, Phys. Rev. D 85, 114006 (2012).

22. X.D. Ji, X. Xiong and F. Yuan, Phys. Rev. Lett. 109, 152005 (2012).

23. C. Lorcé, Phys. Lett. B 719, 185 (2013).

24. R.L. Jaffe and A. Manohar, Nucl. Phys. B 337, 509 (1990).

25. X.D. Ji, Phys. Rev. Lett. 78, 610 (1997).

26. M. Burkardt, Phys. Rev. D 88, 014014 (2013).

27. E. Leader and C. Lorcé, Phys. Rept. 541, 163 (2014).

28. M. Wakamatsu, Int. J. Mod. Phys. A 29, 1430012 (2014).

29. C. Lorcé, Phys. Lett. B 735, 344 (2014).

30. B.U. Musch, P. Hägler, J.W. Negele and A. Schäfer, Phys. Rev. D 83, 094507 (2011).

31. B.U. Musch, P. Hägler, M. Engelhardt, J.W. Negele and A. Schäfer, Phys. Rev. D 85, 094510 (2012).

32. X.D. Ji, Phys. Rev. Lett. 110, 262002 (2013). 